Ann. Génét. Sél. anim., I974, 6 (2), I95-210.

\title{
ONTOGENESE DES MARQUEURS GÉNÉTIQUES SANGUINS CHEZ LE CHEVAL
}

\author{
Marie KAMINSKI, Y. BOUQUET* \\ A. VAN de WEGHE* et Luba PODLIACHOUK** \\ avec la collaboration technique de R. Beaud, F. Pigache et M. Sykiotis \\ Laboratoire d'Enzymologie, C. N. R. S., \\ 91190 Gif sur Yvette \\ * Faculté de Médecine vétérinaire, \\ 9220 Merelbeke (Belgique) \\ ** Centre de Biologie et de Pathologie infectieuse des Équidés, \\ Institut Pasteur, Paris
}

\section{RÉSUMÉ}

La comparaison des antigènes érythrocytaires, de phénotypes des constituants du sérum : préalbumines, albumine, carboxylestérase, transferrine, et des enzymes intra-érythrocytaires : phosphatase acide, catalase, anhydrase carbonique, phosphohexose-isomérase, phosphoglucomutase et 6-phosphogluconate-deshydrogénase, tous présentant le polymorphisme à ]'électrophorèse, ainsi que la démonstration de la $\alpha$-fétoprotéine ont été faites sur du sang des embryons et fœtus entre 6 semaines et I I mois de gestation, des poulains nouveau-nés, normaux, prématurés ou retardés, des jeunes poulains et de leurs parents.

Il a pu être établi que chez le fœtus de cheval on ne trouve pas la carboxylestérase, les préalbumines $\mathrm{X}_{c}, \mathrm{X}_{d}, \mathrm{X}_{h}$ et $\mathrm{X}_{k}$, ni l'activité de l'anhydrase carbonique (la protéine étant présente). Les préalbumines $\mathrm{Pr}$, la " slow $\alpha_{2}$ " globuline, et, exceptionnellement, la carboxylestérase, sont présentes, mais diffèrent qualitativement de leurs homologues adultes. Les constituants suivants sont présents aussi bien dans le sang fœtal que chez l'adulte mais en quantités plus faibles : antigènes érythrocytaires, albumine, transferrine, catalase, phosphatase acide, phosphohexoseisomérase, phosphoglucomutase, 6-phosphogluconate-deshydrogénase.

Il est conclu qu'il est possible d'identifier un poulain à l'aide de ses marqueurs sanguins à partir de l'âge de 3-4 mois sans risques d'erreur.

\section{INTRODUCTION}

Les variations ontogénétiques de constituants sanguins des mammifères que l'on observe entre la période pré- et post-natale dépendent, en dehors du degré d'évolution de l'espèce considérée, de la structure anatomique du placenta. Si la 
barrière placentaire est perméable, le sang fœtal peut, théoriquement, être plus diversifié que celui du nouveau-né. Chez le Cheval, le tissu placentaire est imperméable à la circulation du sang maternel. La comparaison des constituants du sang fœtal de celui du nouveau-né et du jeune poulain révèle ainsi les changements provenant véritablement des processus ontogénétiques et de leur régulation. Comme les résultats ne peuvent être suivis sur un même animal, le nombre d'échantillons examinés doit être élevé pour pallier les éventuelles différences individuelles.

Dans un travail antérieur (KAMINSKI et al., 1970) nous avons décrit l'ontogenèse de la carboxylase sérique sur un matériel assez restreint, surtout en ce qui concerne les échantillons d'âge fœetal. Le matériel étudié dans le présent travail est beaucoup plus vaste. D'autre part, le sang fœtal provient des cas courants de juments gravides et se rapproche davantage des conditions physiologiques normales que le matériel étudié précédemment.

De plus, alors que précédemment nous nous sommes limités à la constatation de la présence ou d'absence d'un constituant, nous avons, dans le travail présent, suivi l'évolution des marqueurs génétiques. Les marqueurs choisis étaient d'une part les groupes sanguins et d'autre part une série de protéines et enzymes sériques ou intra-érythrocytaires, présentant un polymorphisme décelable par électrophorèse. L'étude de ces derniers systèmes offre de plus l'avantage de permettre de suivre les particularités structurales modifiées éventuellement durant l'ontogenèse.

\section{MATÉRIEL,}

Les échantillons étudiés provenaient de 3 origines :

Io Enquête sur les gestations gémellaires chez la jument, conduite par M. VANDEPLASSche (Gand, Belgique) et leur analyse sérologique (groupes sanguins) conduite par L. PodliACHOUK (Paris), I965-1973; jusqu'à présent, 82 cas ont été examinés. Ce matériel comprenait d'habitude le sang des produits et de leurs parents.

$2^{\circ}$ Échantillons de sang de fœtus prélevés sur des juments lors de l'abattage, aux abattoirs de Vaugirard, grâce à la coopération de J. Rigovlet. Le sang de la jument accompagnait celui du fœtus.

$3^{\circ}$ Étude du développement post-natal du poulain, menée en collaboration avec O. GIRARD et J. P. Desormeau du Département des Sérums, Garches, annexe de l'Institut Pasteur. Depuis I 969 nous avons examiné 52 familles.

- Ainsi le sang foetal était de deux catégories :

a) échantillons des abattoirs, qui étaient prélevés stérilement par ponction cardiaque (petits fœtus) ou de la jugulaire (grands fœtus) aussitôt après l'abattage de la jument. L'âge présumé des foetus variait entre 6 semaines et I I mois ; il était déterminé selon la longueur du corps, avec la précision d'environ I mois.

b) fœtus avortés spontanément ou par intervention du vétérinaire lors d'une grossesse gémellaire ; ces foetus étaient souvent momifiés ou mort-nés ; dans certains cas le sang pouvait être pris sur un fœetus encore frais, mais dans la plupart des cas l'hémolyse était très prononcée. La majorité des cas correspond à 7-Io mois et demi de gestation; 3 cas seulement sont de 5 et 6 mois. L'âge de ces fœtus a été déterminé très exactement. gories:

- Échantillons de sang des nouveau-nés et jeunes poulains étaient également de deux caté-

a) gestations gémellaires qui ont fourni des poulains prématurés, mort-nés ou poulains vivants nés après terme, ainsi qu'un cas de superfœtation. Dans cette catégorie nous classons aussi les poulains jumeaux vivants âgés entre 5 et 30 jours. 


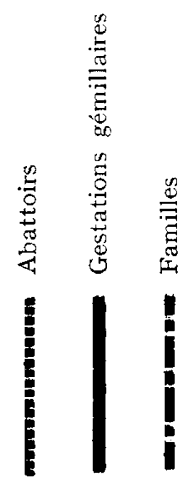

$\frac{0}{0}$
$\frac{0}{5}$
$\frac{1}{0}$
$\frac{0}{0}$
$\frac{0}{2}$
0

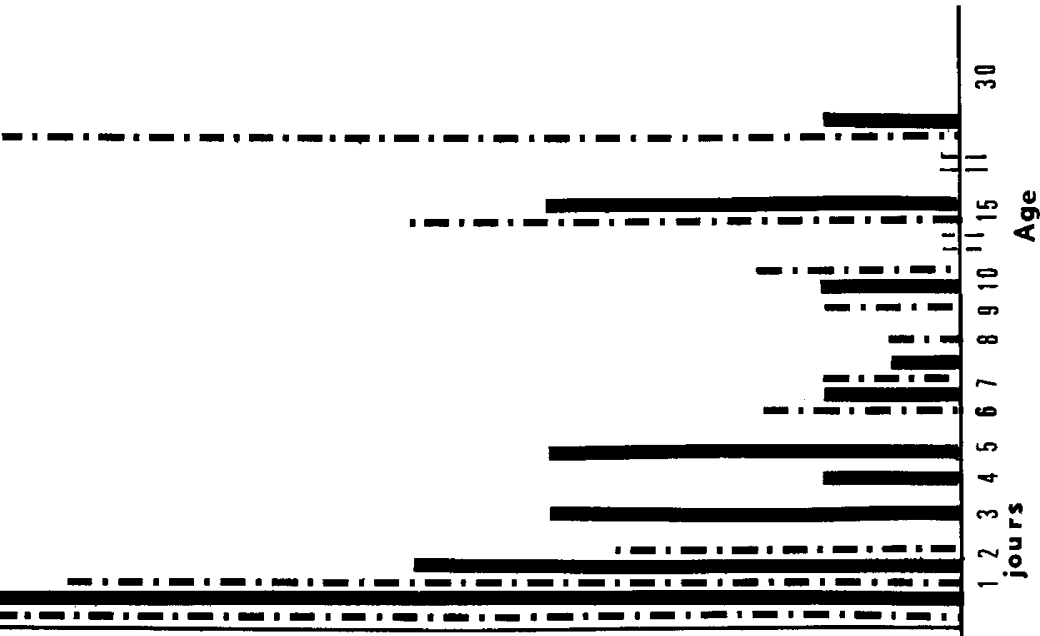

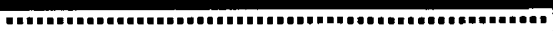

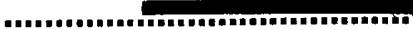

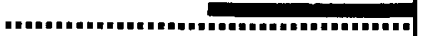

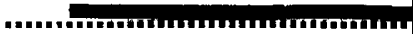

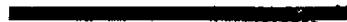
crorrumium

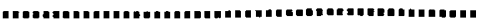
.1.1...

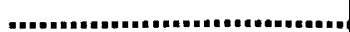
I.and $-1$.

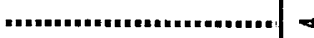

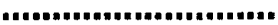
...1.. m

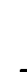

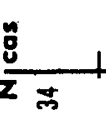
H

r

10


b) séries familiales, comprenant le sang de la jument prélevé le jour de la parturition, le sang du poulain depuis le jour de sa naissance jusqu'à quelques semaines ou mois, le colostrum et quelques échantillons de lait. Le sang et le lait de la jument étaient prélevés, comme le sang du poulain, plusieurs fois à intervalles divers.

La répartition des échantillons est présentée dans la figure $\mathbf{I}$.

\section{MÉTHODES}

\section{Détermination de groupes sanguins}

Le tableau I présente les systèmes des groupes sanguins et tous les 26 facteurs détectés au cours du présent travail. Les I6 facteurs désignés par des lettres majuscules, certaines avec des indices, correspondent aux réactifs (antisérums) obtenus par au moins deux laboratoires différents et contrôlés lors des tests internationaux de comparaison. En dehors de ces réactifs, des laboratoires utilisent leurs réactifs propres, qui déterminent des facteurs désignés soit par des lettres majuscules, F, G et $\mathrm{P}_{2}$ obtenus à l'Institut Pasteur, soit par des chiffres arabes (dans l'ordre chronologique de découverte), précédés par le code du laboratoire (initiales du pays et de la ville).

TABLEAU I

Antigènes érythrocytaires chez le Cheval

\begin{tabular}{|c|c|c|c|c|c|c|c|c|c|}
\hline Système & A & $\mathrm{C}$ & & $\mathrm{D}$ & $\mathbf{K}$ & $\mathbf{P}$ & $Q$ & $\mathrm{U}$ & ? \\
\hline $\begin{array}{c}\text { Antigènes } \\
\text { érythr. }\end{array}$ & $\begin{array}{c}A_{1} \\
F \\
H \\
A^{\prime} \\
F / P^{*} 17\end{array}$ & C & $\begin{array}{c}D \\
E_{1} \\
E_{2} \\
E^{\prime} \\
F / P 8\end{array}$ & $\begin{array}{c}F / P 14 \\
J_{1} \\
J_{2} \\
G \\
Y\end{array}$ & $\begin{array}{l}\mathrm{K} \\
.\end{array}$ & $\begin{array}{c}\mathrm{P}_{1} \\
\mathrm{P}_{2} \\
\mathrm{~F} / \mathrm{P} 9 \\
\mathrm{P}^{\prime}\end{array}$ & $\frac{\mathrm{Q}}{\mathrm{F} / \mathrm{P} 10}$ & $\mathrm{U}$ & $\begin{array}{l}\mathrm{F} / \mathrm{P} 12 \\
\mathrm{~F} / \mathrm{P} 18\end{array}$ \\
\hline
\end{tabular}

* $\mathrm{F} / \mathrm{P}=$ France $/$ Paris.

Les déterminations des antigènes érythrocytaires ont été faites soit à l'aide de la réaction d'agglutination, soit à l'aide de la réaction d'hémolyse.

Les recherches sur les groupes sanguins chez les jumeaux du cheval s'étendent sur une période de 8 ans ; ce matériel était l'unique source de sang fœtal jusqu'en I973 où nous avions reçu la série d'échantillons des abattoirs. Durant la période entre I965 et 1973, la recherche sur les groupes sanguins chez le Cheval, menée à l'Institut Pasteur, a permis de passer de 15 facteurs à 26. Ainsi le nombre de réactifs disponibles (antisérums) permettant de déterminer un facteur particulier dans le travail présent dépend de la date à laquelle l'analyse avait été exécutée.

\section{Détermination de phénotypes des protéines et enzymes polymorphiques}

Les variants électrophorétiques des protéines sanguines ont été détectés par électrophorèse horizontale en gel d'amidon, en utilisant les techniques particulières à chaque système.

\section{Protéines sériques.}

\section{Préalbumines : Pr.}

La technique originale de BRAEND (1970) a été modifiée : le tampon $\mathrm{pH}$ 6,3 est utilisé pour les bacs, à la molarité égale à la moitié de celle décrite par BRAEND, et pH 5 pour le gel. Les plaques sont refroidies à $6^{\circ} \mathrm{C}$ en utilisant une enveloppe liquide en antigel. Avec $300 \mathrm{~V}$ la distance de migration est de $9 \mathrm{~cm}$ après $6 \mathrm{~h}$. 


\section{Albumine: Al.}

La méthode utilisée est de Ashron et Lampkin (I965) basée sur celle de Stormont et Suzuki (1963). Une technique légèrement différente a été également utilisée : le tampon borate de $\mathrm{Na}$ $\mathrm{pH}$ 8,5 dans les bacs et $0,004 \mathrm{M}$ Tris-o,oo8 M citrate $\mathrm{pH} \mathrm{5,4}$ pour le gel. L'électrophorèse est faite avec $30 \mathrm{~mA}$ et $80 \mathrm{~V}$ par plaque, jusqu'à ce que la tache de Bleu de bromophénol atteigne I $2 \mathrm{~cm}$ depuis l'origine.

\section{Transferrine: $T f$.}

Le tampon pour le gel est 0,072 M Tris-o,o06 M citrate $\mathrm{pH} \mathrm{8,7,} \mathrm{mélangé} \mathrm{en} \mathrm{proportions}$ 5,5 à I avec le borate de $\mathrm{Li}$ ( $\mathrm{LiOH}$ o,05 $\mathrm{M}$ et o, I9 $\mathrm{M}$ acide borique). Dans les bacs le tampon $\mathrm{Li}$ est utilisé seul. Le courant est initialement $300 \mathrm{~V}$ pendant ro mn et ensuite i $80 \mathrm{~V}$; la migration est faite à la température de laboratoire.

Ces plaques sont découpées en deux couches, l'une révélée pour la carboxylestérase, l'autre colorée par le Noir Amide. Cette dernière montre, en plus des bandes de transferrine, des variations de la bande de "slow $\alpha_{2}$ " et, dans les sérums fœetaux, une bande intense dans la région des postalbumines, identifiée comme la $\alpha$-fétoprotéine.

4. Estérase: Es. (Carboxylestérase E.C. 3.I.I.I.).

Deux méthodes ont été utilisées, l'une à pH 8,7, selon GAHNE (I966), l'autre à pH 4,5 d'après ScotT (I972). La première méthode permet de distinguer, en utilisant comme substrat l'acétate de $\beta$-naphtyle, les bandes de $E_{2}$ (cholinestérase), $E_{3}$ et les taches de $E_{4}$ et $E_{5}$, et, suivant le cas, la série de bandes d'isozymes dans la région des $\beta$-globulines (KAMINski et al., I970). Avec l'acétate de $\alpha$-naphtyle comme substrat on détecte seulement les zones $E_{2}$ et $E_{5}$. Dans cette dernière zone est visible un polymorphisme décrit par GAHNE, comportant 6 phénotypes résultant des combinaisons de 3 allèles. L'absence d'activité dans cette zone peut être due soit à un gène récessif (GAHNE, r966), soit au manque de synthèse, caractéristique de la période foetale (KAMINSKI et al., I970), soit enfin, chez d'autres Équidés, au manque probable du gène lui-même (KAMINSKI, ig69; Kaminski et Podliachouk, r970).

Dans la seconde méthode il n'y a pas de différence entre les deux substrats car seule la $E_{5}$ (carboxylestérase) est détectée. Son polymorphisme est plus varié qu'à pH alcalin : nous avons observé à ce jour 9 phénotypes différents, correspondant aux 5 allèles.

L'immunoélectrophorèse a été faite comme précédemment (KAMINSKI, I969), en utilisant des antisérums du Lapin anti-sérum de cheval adulte ou foetal. L'arc de la carboxylestérase n'est pas visible en tant que précipité antigène-anticorps, mais seulement après révélation de l'activité enzymatique.

\section{5. $\alpha$-fétoprotéine.}

Ce constituant des sérums fœtaux a été détecté soit par électrophorèse en gel d'amidon à $\mathrm{pH} 8,7$ et coloration des protéines, soit par immunoélectrophorèse avec l'antisérum anti-sérum fœtal.

\section{Enzymes intra-érythrocytaires.}

Les phénotypes de catalase, anhydrase carbonique, phosphatase-acide, phosphohexoseisomérase, phosphoglucomutase et 6-phospho gluconate-deshydrogénase ont été déterminés après électrophorèse des hémolysats. Ceux-ci ont été préparés à partir des érythrocytes frais, lavés et centrifugés à 3 reprises, ensuite congelés et décongelés de nouveau à 3 reprises, finalement centrifugés. Le surnageant doit être utilisé immédiatement.

L'activité péroxydasique de la catalase, (Cat., $\mathrm{H}_{2} \mathrm{O}_{2}: \mathrm{H}_{2} \mathrm{O}_{2}$ oxydoréductase E. C. I.I r.r.6.) est démontrée selon KELly et al. (I97I) sur des gels imbibés de Iodure de potassium.

L'anhydrase carbonique, (CA, carbonate hydrolyase, E.C. 5.3.I.9.) est révélée selon SANDBERG (I968). Afin d'améliorer la résolution des bandes rapides, on extrait au préalable l'hémoglobine avec l'éthanol-chloroforme.

L'activité de la phosphatase acide, (AP, orthophosphoric monoester phosphohydrolase, E.C. 3.I.3.2.), est révélée selon Hopkinson et al. (I963); la phosphohexose-isomérase, (PHI, D-glucose-6-phosphate ketol isomérase, E.C. 5.3.1.9.) et phosphoglucomutase, (PGM $\alpha$-Dglucose I-6-diphosphate $\alpha$-D-glucose-I-phosphate phospho-transférase, E.C. 2.7.5.I.), sont révélées selon SPENCER et al. (I964) et DETTER et al. (I968); la 6-phosphogluconate deshydrogénase, (6-PGD, 6-phospho-D-gluconate : NADP oxydoréductase, E.C. I.I.I.44.) est révélée d'après FILDES et PARR (I963), technique adaptée au cheval par SANDBERG et BENGTSSON I970).

Il est possible de démontrer simultanément (sur une même plaque de gel) les activités AP, PHI, PGM et 6-PGD suivant BENGTSSon et SANDBERG (1973). L'électrophorèse est faite soit 
durant 5 heures avec $\mathrm{I}_{5} \mathrm{~V} / \mathrm{cm}$, soit avec $5 \mathrm{~V} / \mathrm{cm}$ pendant $\mathrm{I} 6 \mathrm{~h}$, ce qui n'exige pas de réfrigération autre que l'installation dans une chambre froide à $+4^{\circ} \mathrm{C}$. Trois tranches sont découpées du côté anodique de la plaque et une du côté cathodique ; elles sont révélées pour AP, 6-PGD et PGM qui migrent vers l'anode et PHI qui migre vers la cathode.

Les enzymes glycolytiques sont tous révélés par la réduction du sel de tétrazolium MTT et la formation d'un précipité bleu de formazan par l'intermédiaire de phénazine-méthosulfate (PMS).

\section{RÉSULTATS ET DISCUSSION}

Les antigènes érythrocytaires et les protéinogrammes du sérum ont été déterminés sur la totalité du matériel disponible (avec la restriction mentionnée plus haut concernant le nombre des antigènes, suivant l'année). Les enzymes intra-érythrocytaires ont été déterminés uniquement sur une partie des échantillons de sang fœtal et celui des juments des abattoirs.

\section{Antigènes érythrocytaires et les anticorps naturels}

Pour les 3 embryons : 6 semaines, 2 et 3 mois, provenant des abattoirs, quelquesuns des facteurs ont fourni des réactions plus faibles (lues à ++ au lieu de ++++ ) que celles des érythrocytes des adultes (mères). D'autre part, 4 embryons âgés d'environ 3 mois ont fourni, pour tous les antigènes décelés (2I sur 26), des réactions d'hémolyse ou d'agglutination comparables en intensité avec celles des adultes. Les 5 facteurs restants, non décelés, sont tous des facteurs rares. Cependant, même ces facteurs rares ont été trouvés chez les fotus plus âgés : soit $\mathrm{E}_{1}, \mathrm{H}$ et $\mathrm{J}_{1}$ chez les foetus de $4-5$ mois; $\mathrm{Y}$ et $\mathrm{K}$ chez ceux de 7 mois. L'intensité des réactions était égale à celle des adultes.

Ainsi, certains antigènes érythrocytaires sont déjà décelables chez le cheval dès la période embryonnaire ; tout au plus on peut dire que les réactions sont plus faibles. La présence de facteurs érythrocytaires durant la période fœetale a été démontrée chez d'autres espèces de mammifères (ShAw et STONE, I962).

La présence d'isoagglutinines a été recherchée chez une vingtaine de fœetus âgés entre 4 et Io mois, qui étaient négatifs pour les facteurs $\mathrm{A}$ ou $\mathrm{C}$ : on n'a pas pu démontrer des anticorps correspondants. Chez les adultes ne possédant pas ces facteurs, $80 \mathrm{p}$. Ioo des sérums contiennent les anticorps. Nos résultats négatifs sont vraisemblablement imputables à la déficience fotale en immunoglobulines, pas encore synthétisées par le fotus et dont le transfert à partir du sang maternel est empêché par la structure du placenta.

\section{Variants électrophorétiques des protéines sériques}

\section{Préalbumines.}

Ce groupe de protéines, principalement glycoprotéines douées d'activités variées, tant biochimiques que physiologiques, est très hétérogène. Des variants électrophorétiques ont été observés, permettant de distinguer 5 systèmes que l'on désigne, dans l'ordre décroissant de migration anodique, $\mathrm{Pr}, \mathrm{X}_{c}, \mathrm{X}_{d}, \mathrm{X}_{h}$ et $\mathrm{X}_{k}$. Des bases génétiques de transmission ont pu être établies pour les seuls systèmes $\operatorname{Pr}$ et $\mathrm{X}_{k}$ (GAHNE, I966; BRAEND, I970). 
Les sérums des adultes et fotaux présentent des différences dans les systèmes suivants :

- système $\operatorname{Pr}$ : les juments gravides examinées ont montré 8 phénotypes, parmi lesquels les plus fréquents étaient LL et SS, alors que leurs fœetus n'ont pas formé de bandes L ni S. Tous les sérums fœetaux ont montré le même protéinogramme, composé de bandes faibles et d'une zone large migrant plus rapidement que les bandes les plus anodiques chez l'adulte. Ces constituants n'ont pas été observés dans aucun sérum d'adulte examiné.

- Les systèmes $\mathrm{X}_{c}, \mathrm{X}_{d}$ et $\mathrm{X}_{h}$, ressemblant au système $\mathrm{Pi}$ (anti-trypsine) de sérum humain (FAGERHOL, I967), étaient pratiquement absents des sérums fœetaux ; lorsqu'il y avait des bandes dans cette zone, elles étaient faibles et ne correspondaient pas aux zones connues chez l'adulte.

- Le système $\mathrm{X}_{k}$ n'a pa être détecté du tout dans les sérums fœetaux avant 9 mois. Parmi les nouveau-nés et jeunes poulains plusieurs étaient négatifs pour $\mathrm{X}_{k}$; les bandes commençaient à être visibles chez les poulains d'une semaine, et chez certains, seulement à 3 semaines (fig. $2 a$ ).

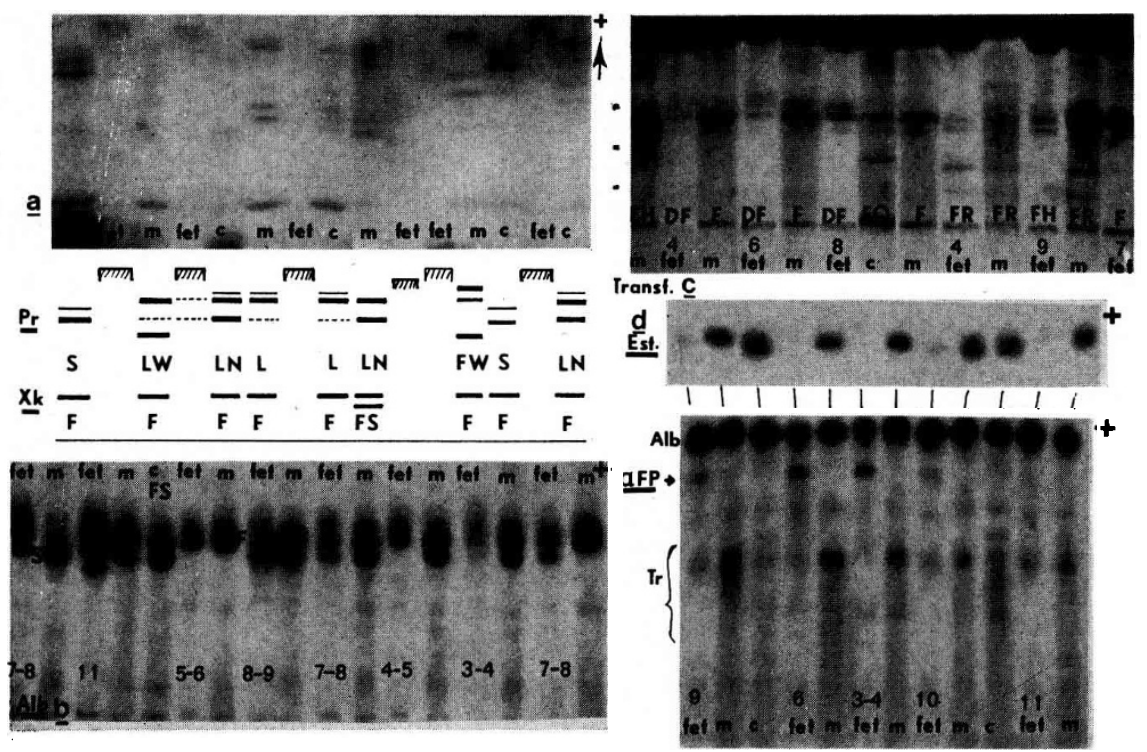

Fig. 2. - Protéinogrammes et zymogrammes en gel d'amidon des sérums foéaux et des juments

a : Zone des préalbumines $\operatorname{Pr}$ et $\mathrm{X}_{k}$. Différences qualitatives entre les sérums foetaux et adultes.

b : Phénotypes d'albumine; différences quantitatives entre les sérums fœtaux et adultes.

c: Phénotypes de transferrine; différences quantitatives et phénotypes à 4 bandes chez le fœtus.

d-e : Deux tranches d'un même gel, révélées pour l'estérase (en haut) et les protéines (en bas). Absence d'estérase ou traces d'activité chez les fœtus les plus âgés. Présence de la $\alpha$-féroprotéine et la corré lation avec l'absence d'estérase.

\section{Albumine.}

Seules les deux bandes principales, $\mathrm{F}$ et $\mathrm{S}$, ont été détectées. Les phénotypes maternels et fœtaux étaient comparables quant à la position des bandes, mais l'intensité de coloration était plus faible chez les seconds (fig. $2 b$ ). 


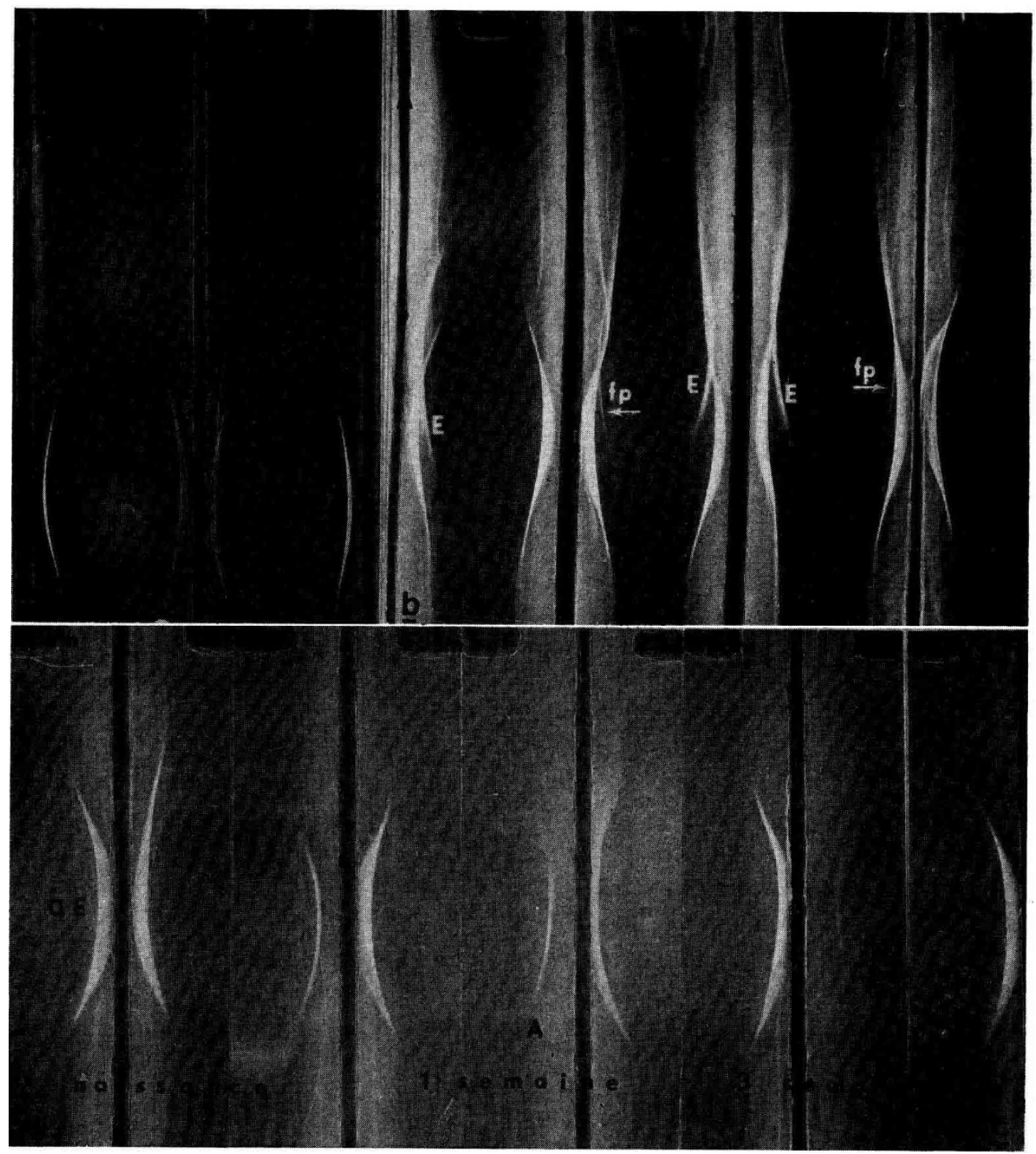

FIG. 3. - Démonstration immunoélectrophorétique de la $\alpha$-fétoprotéine

a : Avec l'immunsérum anti-sérum fœtal. A désigne albumine.

b : Famille avec des jumeaux nouveau-nés : la plaque d'immunoélectrophorèse a été d'abord révélée pour l'activité estérasique et ensuite colorée pour les protéines; de cette façon la $\alpha_{1}$-carboxylestérase et la $\alpha$-fétoprotéine sont visibles toutes les deux. L'estérase est présente dans tous les sérums, mais n'est révélée qu'avec l'immunsérum anti-sérum de cheval adulte ; la $\alpha$-fétoprotéine est absente chez les adultes et n'est révélée qu'avec l'immunsérum anti-sérum fœtal.

c : Démonstration immunoélectrophorétique de la progression inverse de l'activité estérasique dans le sérum du poulain et dans le colostrum et lait maternels. 


\section{3. $\alpha$-fétoprotéine.}

Les sérums fotaux ont présenté une bande intense qui suit l'albumine. Cette bande est absente dans les sérums des juments mères (fig. $2 e$ ).

En immunoélectrophorèse on détecte une ligne dans la zone des $\alpha$-globulines, présente dans les sérums de fœtus mais absente chez les adultes. Cette ligne n'est pas formée si on utilise l'immunsérum anti-sérum de cheval adulte (fig. $3 a$ et $b$ ).

\section{Transferrine.}

Parmi les 6 bandes connues, seule la bande $M$ n'a pas été rencontrée dans notre matériel. Les phénotypes des sérums fœtaux peuvent être déterminés, bien que les bandes soient plus faibles que chez les adultes. Chez les fotus les plus jeunes, les types hétérozygotes composés de bandes $\mathrm{D}, \mathrm{F}$ et $\mathrm{H}$ ont paru plus complexes que chez les chevaux adultes (fig. $2 c$ ).

\section{Estérases.}

a) Carboxylestérase $\left(\alpha_{1}, \mathrm{E}_{5}\right)$.

La plupart des sérums fœtaux ne contiennent pas d'estérase confirmant ainsi les conclusions du travail précédent (KAminski et al., I970).

Cependant nous avons rencontré quelques exceptions à cette règle :

- Trois fotus d'environ 9 mois provenant du groupe des Abattoirs ont montré des traces d'activité ; chez ces sérums une ou deux bandes seulement étaient visibles, au lieu de phénotype complet. Deux autres fœetus du même groupe ont fourni des zymogrammes ressemblant aux phénotypes des adultes, mais très faibles. Ennfin, un fœtus à terme et un âgé de 9 mois ont formé des zymogrammes composés de 4 bandes, qui ne pouvaient pas être interprétées en termes de phénotypes connus - en effet, aucune des bandes principales ne correspondait aux bandes du zymogramme maternel (fig. $4 c$ ).

- Parmi les fœetus du groupe des jumeaux, la plupart ne présentaient pas d'activité estérasique. Chez une paire de jumeaux de 9 mois ainsi que dans 3 sérums correspondant à Io mois et demi on a relevé une bande d'activité. Une paire de jumeaux a formé des zymogrammes de 4 bandes ou une seule des bandes principales correspondait aux bandes des parents. Finalement, une paire des jumeaux à terme a formé des zymogrammes différents entre eux mais tous les deux interprétables (fig. $4 b$ et $d$ ).

En dehors de ces cas étudiés en gel d'amidon, un seul sérum fœetal, correspondant à 7 mois, a formé un arc d'activité estérasique en immunoélectrophorèse.

Pour les sérums de nouveau-nés, l'activité estérasique est très faible; les deux groupes d'échantillons ont donné des résultats similaires (fig. $4 e, g$ et $h$ ).

Lorsque des traces étaient détectées le phénotype n'était pas complet - ceci est probablement dû à une différence d'ordre quantitatif. Il faut remarquer que les mêmes sérums examinés à $\mathrm{pH} 8,5$ étaient plus faciles à analyser ; aussi en immunoélectrophorèse a été observé un arc d'estérase, bien que petit et faible.

Il faut souligner que les diverses étapes de l'ontogenèse de la carboxylestérase varient beaucoup selon le cas individuel. Ainsi nous avons rencontré quelques sérums de poulains âgés de 2-3 jours qui étaient encore négatifs pour l'estérase, alors que d'autres poulains présentaient déjà des phénotypes distincts à I jour. La biosynthèse 

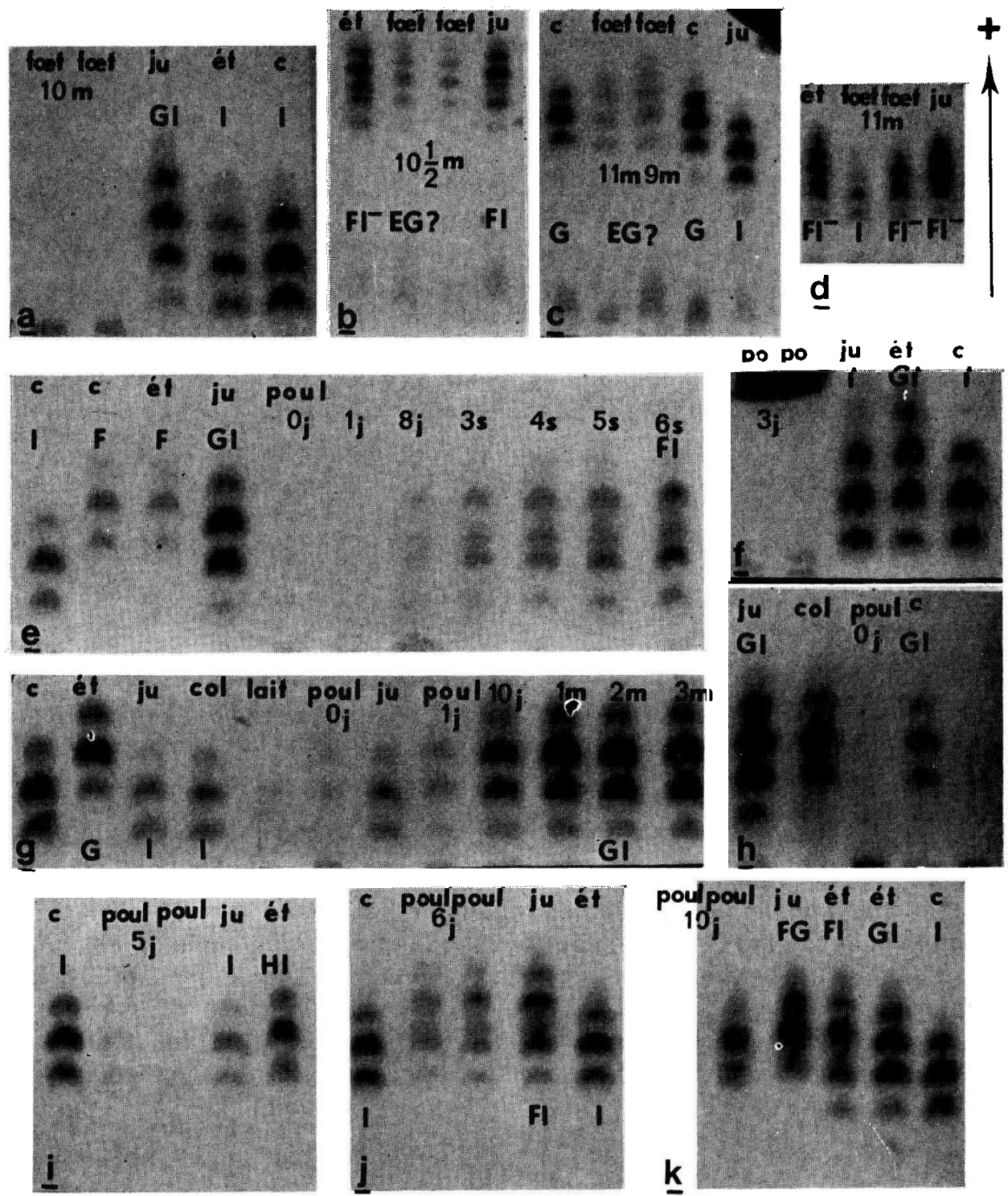

${ }_{0}$ oul ju pouli
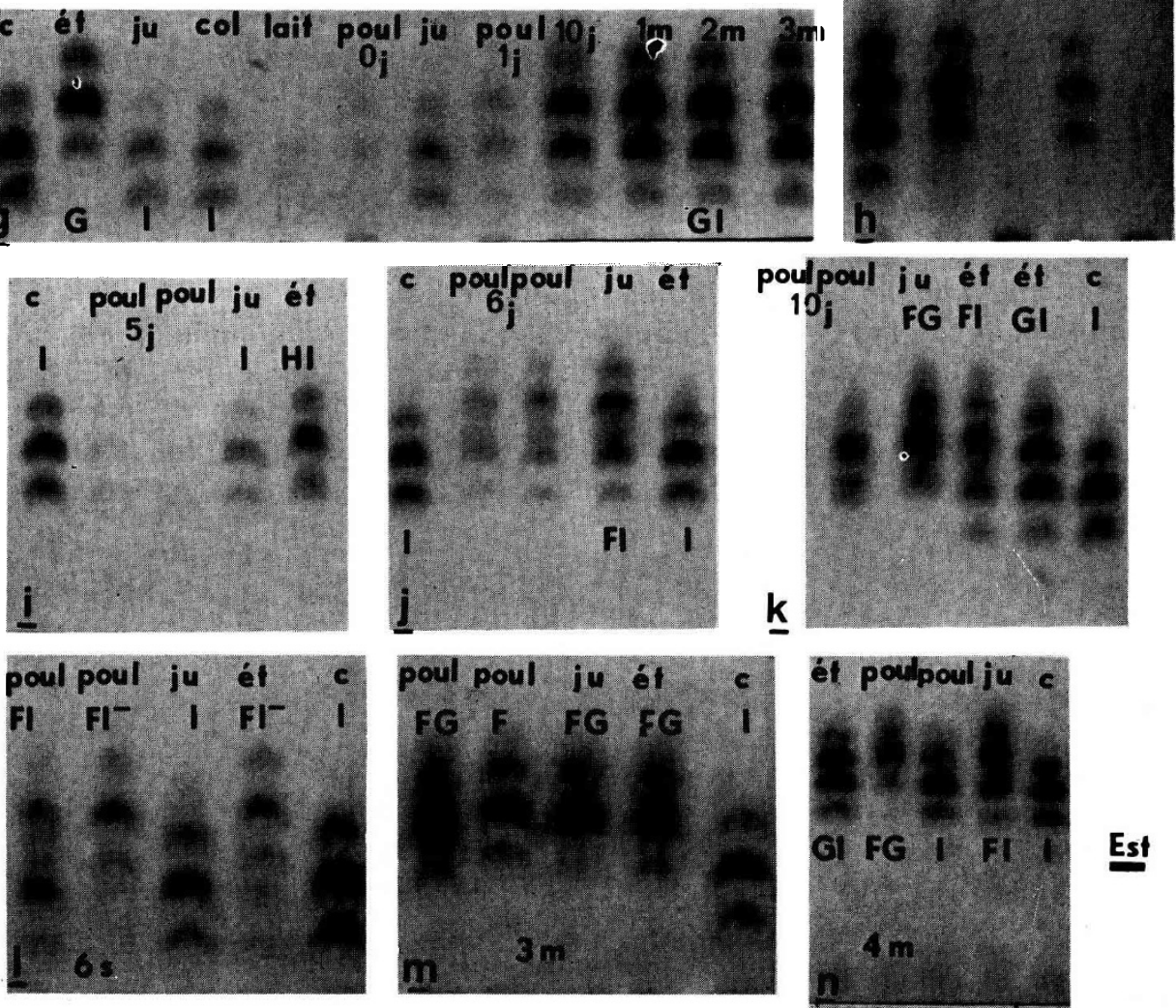

Est 
de cet enzyme pourrait dépendre de facteurs du milieu, ou bien sa régulation ne serait pas très stricte (fig. $4 f$ et $g$ ).

Durant les premiers jours les poulains forment en général des phénotypes faibles et souvent incomplets, mais à partir d'une semaine ceux-ci peuvent être déterminés. Dans quelques échantillons les zymogrammes étaient légèrement décalés par rapport à ceux des adultes (fig. $4 i, j$ et $k$ ).

La comparaison de zymogrammes de plusieurs échantillons de sérum du même animal prélevés durant sa croissance montre des changements d'ordre quantitatif (fig. $4 e$ et $g$ ). Cette variation quantitative déjà reportée (KAMINSKI et al., I970), aboutit, généralement aux environs d'un mois, à un taux similaire à celui des adultes.

Il faut remarquer ici que les taux d'estérase varient parmi les sérums d'adultes. Cela peut être imputable soit à la présence d'allèle " silencieux " $E s^{0}$ (GAHNE, I966), soit à la régulation métabolique. Des dosages d'activité durant le processus ontogénétique ne pourraient être correctement interprétés sans la connaissance des causes de la variation chez les adultes.

La transmission des allèles $F, G$ et $I$ a été vérifiée chez des familles : elle suit les lois de Mendel. Dans les cas des familles avec des jumeaux, bien que tous soient dizygotiques (PODLIACHOUk et al., I974), nous avons trouvé autant de paires présentant des phénotypes identiques que de différents (fig. $4 j, k, m$ et $n$ ).

La figure $4 l$ présente une famille où la jument est de type I, l'étalon de type FI " déficient" - dans ce zymogramme la bande principale I est très faible désigné $F I^{-}$(KAminski, résultats non publiés). Les deux jumeaux présentent le phénotype FI, l'un normal, l'autre déficient. Le gène $I$ chez les produits venant obligatoirement de la mère, on peut supposer :

FIG. 4. -- Zymogrammes en gel d'amidon à pH 4,5 de l'estérase chez les foetus, nouveau-nés et jeunes poulains

Les âges sont indiqués en jours, semaines et mois

a : Fœtus jumeaux sans activité estérasique.

b : Fœtus jumeaux montrant des phénotypes non compatibles avec ceux des parents; forme fœtale de l'estérase supposée.

c : Deux fœtus, chacun ayant le phénotype non compatible avec celui de sa mère.

$\mathrm{d}:$ Fœtus à terme (jumeaux), phénotypes compatibles avec ceux des parents.

e : Progression de l'estérase chez un poulain : augmentation de l'activité sans changement de phénotype.

$f$ : Poulains jumeaux dépourvus d'activité estérasique.

g : Progression de l'estérase chez un poulain, comparaison avec le colostrum et le lait.

h : Zymogramme du colostrum, comparé au sérum de la jument.

i : Zymogrammes incomplets et activité faible chez des poulains jumeaux de 5 jours.

$\mathrm{j}$ : Phénotypes complets, identiques, mais faibles, chez des jumeaux dizygotiques de 6 jours.

k: Phénotypes identiques à l'un des parents mais un peu plus lents; un cas de paternité douteuse : les deux étalons apparaissent compatibles.

1: Famille avec jumeaux ; sur la base des phénotypes parentaux l'un des poulains est déterminé comme $\mathrm{FI}^{-}$, bien que le zymogramme apparaisse comme $\mathrm{F}$, ce qui correspondrait à un homozygote.

$\mathrm{m}$ : Phénotypes différents chez des jumeaux dont les parents sont identiques.

$\mathrm{n}$ : Phénotypes hétéro- et homozygotes chez des jumeaux dont les parents, tous les deux hétérozygotes, sont différents.

Les conditions d'électrophorèse ont varié d'une plaque à l'autre mais toutes les plaques étaient découpées à la ligne d'origine. 
- soit I : que le génotype de celle-ci est $I$ normal/I déficient où le $I$ - n'est pas perceptible dans nos conditions expérimentales ;

- soit 2 : que la formation du $I$ - résulte des effets de régulation du gène $I$.

Concernant l'ontogenèse de la carboxylesterase en général, nous devons considérer d'une part sa présence et d'autre part le zymogramme obtenu. Sur i Io sérums fœtaux examinés, I4 présentaient quelque activité ; sur ces I4, seuls 4 ont formé des zymogrammes ressemblant à ceux des adultes. Cependant, en se basant sur les lois de transmission, ces 4 cas apparaissaient incompatibles avec des phénotypes parentaux. Ces cas peuvent correspondre à une forme de transition de l'estérase se manifestant à la fin de la période fotale. Cependant, comme nous l'avons dit plus haut, dans la grande majorité des cas examinés la carboxylestérase est absente durant la vie fœtale et apparaît à la naissance.

b) $\alpha_{0}$-estérases.

- un arc observé en immunoélectrophorèse à l'intérieur de celui d'albumine en utilisant l'immunsérum du lapin anti-sérum d'âne était présent seulement chez les adultes et non dans le lait ou colostrum; les sérums de nouveau-nés ont formé cet arc faible. L'un des poulains saigné deux jours plus tard présentait une activité augmentée. Ce constituant réagit seulement sur l'acétate de $\beta$-naphtyle, et non sur celui d' $\alpha$-naphtyle, comme la $\alpha_{1}$ estérase (carboxylestérase).

- dans une autre famille, un autre constituant localisé comme $\alpha_{0}$ a été observé : il réagit avec l'immunsérum anti-cheval adulte et est révélé par l'acétate de $\alpha$-naphtyle; cette estérase était absente du colostrum, lait et sérum du poulain à la naissance. Le lendemain on a pu la détecter chez le poulain.

\section{Variants électrophorétiques des enzymes intra-érythrocytaires}

\section{Catalase.}

Les 3 phénotypes $\mathrm{F}, \mathrm{M}$ et $\mathrm{S}$, correspondant aux génotypes contrôlés par les allèles $F$ et $S$ ont tous été détectés dans les hémolysats tant fœtaux qu'adultes. Aucune différence n'a été constatée selon 1'âge de l'animal (fig. 5).

2. Anhydrase carbonique.

Les juments examinées présentaient seulement les phénotypes I, FI et IO. Parmi les échantillons fœtaux, I3 sur 38 n'ont formé aucune bande dans cette zone et aucune activité enzymatique n'a été décelée. Dans 20 cas on a observé une faible zone de protéine localisée entre les bandes I et $O$. Dans 5 cas on a observé une double zone de protéine, localisée comme précédemment entre I et $O$. Les produits des juments présentant les phénotypes FI ou IO n'ont pas formé de bandes F, I ni O (fig. 5).

Nous n'avons pas établi de relation entre l'âge des fœtus et les zymogrammes formés (ou l'absence de bandes). Il semble bien que l'anhydrase carbonique de type adulte n'existe pas chez les fœtus qui possèdent, de façon irrégulière, une protéine qui serait le précurseur de l'enzyme.

\section{Phosphatase acide.}

Chez les juments nous avons trouvé les phénotypes F, FS et $\mathrm{S}$, alors que chez les fœtus, seuls FS et $S$ ont été observés. Aucune différence n'a été constatée entre les hémolysats des adultes et fœtaux. 

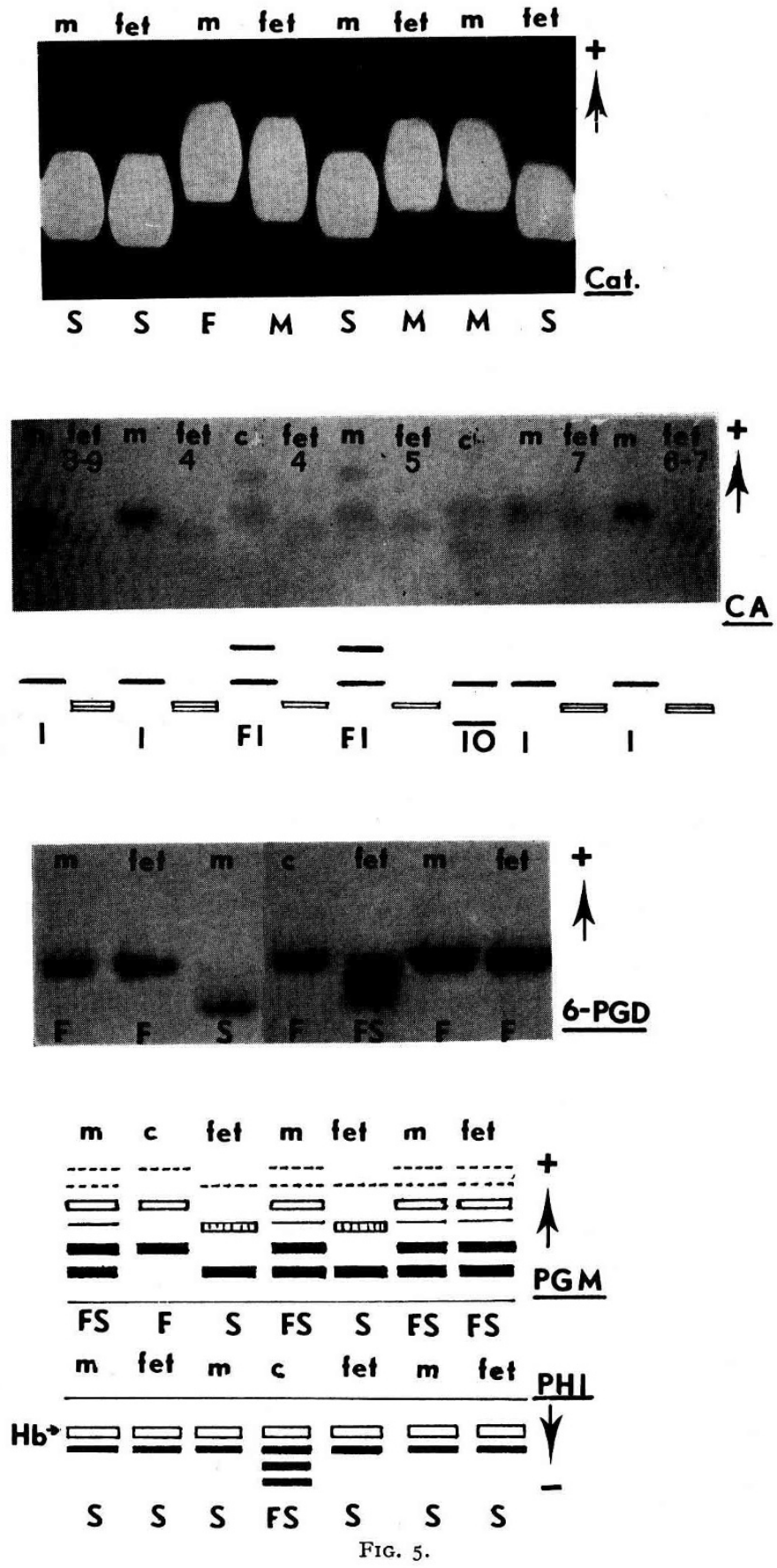


\section{Phosphohexose-isomérase PHM et Phosphoglucomutase PHI.}

Seuls les phénotypes FS et S de PGM ont été trouvés : il n'y avait aucune différence entre les adultes et les foetus.

Pour PHI, tous les échantillons étudiés appartenaient au type S (fig. 5).

\section{6-Phosphogluconate deshydrogénase.}

Tous les phénotypes connus ont été rencontrés dans cette étude, aussi bien chez les adultes que chez les fotus.

\section{DISCUSSION E'T CONCLUSIONS}

Dans une étude ontogénétique comparative, on pourrait s'attendre à ce que les résultats puissent être classés en 3 catégories :

- Constituants typiquement fotaux, n'existant pas chez l'adulte.

- Constituants présents durant toute la vie de l'individu, définis par leur activité antigénétique ou enzymatique ou des propriétés physiques et chimiques ; ces constituants peuvent présenter une variation quantitative suivant l'âge.

- Constituants adultes n'existant pas chez le fœetus.

La présence ou l'absence d'un constituant durant la vie foetale résulte de la convergence de plusieurs mécanismes physiologiques. Pendant des stades précoces du développement d'un organisme supérieur, le rôle primordial est joué par le système vasculaire. On suppose que l'embryon tend à être autonome et indépendant de la mère surtout pour des constituants sanguins assurant les fonctions de transport ainsi que la production d'énergie par la glycolyse dans les érythrocytes, alors que, par exemple, l'équilibre acido-basique ou l'activité anti-trypsine peuvent dépendre de l'apport maternel.

On peut donc s'attendre à ce que l'activité enzymatique et les détails structuraux qui déterminent le polymorphisme génétique soient en place dès le début de l'ontogenèse pour le premier groupe de constituants. Par contre les autres constituants, responsables des activités biologiques dont les manifestations ne sont pas vitales dès le stade fœtal, peuvent être soit absents, soit présents dans une forme différente de celle de l'adulte.

Nos résultats sont en accord avec cette hypothèse en montrant d'une part la similarité de l'albumine, de la transferrine, des enzymes glycolytiques et de la catalase chez le fotus et chez l'adulte, et d'autre part des différences marquées pour les préalbumines, la carboxylestérase et l'anhydrase carbonique.

Du point de vue de la recherche appliquée, telle que le contrôle de filiation ou les problèmes d'identification, nos résultats permettent de proposer 1'âge 3-4 mois comme le stade le plus précoce du développement du poulain où il est possible d'effectuer une détermination définitive de divers systèmes génétiques sanguins étudiés. 


\section{REMERCIEMENTS}

Les auteurs remercient vivement les Professeurs M. VAndeplassche et O. Girard et les Docteurs J.-P. Desormeau et J. Rigoulet qui leur ont fourni aimablement tout le matériel.

Nous remercions, pour l'excellente collaboration technique, Mmes R. Beaud, F. Pigache et Mile M. Sykioris.

Ce travail a bénéficié du support financier de la Société d'Encouragement pour l'Amélioration des races de chevaux en France (Directeur J. RomaneT) et de l'I. R. S. I. A.-I. W. O. N. L. à Bruxelles (Belgique). (Institut pour l'Encouragement de la Recherche Scientifique dans l'Industrie et l'A griculture).

\section{SUMMARY}

\section{DEVELOPMENTAL SEQUENCE OF GENETIC MARKERS IN HORSE BLOOD}

Investigation of series of genetic markers was performed on blood samples from embryos, fœtuses, newborn and young foals; the comparison with adult horse (parents) blood was established.

The genetic systems studied were :

- the erythrocyte antigens (blood groups) ;

- the polymorphic serum components (prealbumins, albumin, carboxylesterase and transferrin) ;

- the polymorphic intra-erythrocyte enzymes (acid phosphatase, carbonic anhydrase, catalase, phosphohexose isomerase, phosphoglucomutase and 6-phosphogluconate dehydrogenase).

Besides these components, the investigation of $\alpha$-fetoprotein and two ill defined esterases was also carried out.

$\alpha$-fetoprotein is the characteristic fotal component, but a few other components may be found in foetal blood, differing qualitatively from their adult counterparts : prealbumin Pr, slow $\alpha_{2}$ globulin (present in all samples), a protein probably corresponding to carbonic anhydrase but devoid of enzymatic activity, and in rare cases the carboxylesterase.

Following components are lacking in fœtal blood : prealbumins $\mathrm{X}_{c}, \mathrm{X}_{d}, \mathrm{X}_{h}$ and $\mathrm{X}_{k}$; carboxylesterase and two other esterases ; besides, there is no component exhibiting the activity of carbonic anhydrase.

Following components are present in fœetal as well as in adult blood, in increasing amounts during the foetal development and postnatal growth : erythrocyte antigens, albumin, transferrin, catalase, acide phosphatase, phosphohexose-isomerase, phosphogluconate dehydrogenase.

The authors propose the age of $3-4$ months as a stage of maturation where the identification of a foal by means of blood genetic markers can be performed safely.

\section{RÉFÉRENCES BIBLIOGRAPHIQUES}

Ashton G. C., LampKin G. H., r965. Serum albumin and transferrin polymorphism in East African cattle. Nature, 205, 209-2ro.

Bengtsson S., SANDBerg K., I973. A method for simultanojus electrophorezis of four horse red cell enzyme systems. Anim. Blood Grps Biochem. Genet., 4, 83-87.

Braend M., I970. Genetics of horse acidic prealbumins. Genstics, 65, 495-503.

Detrer J. C., Ways P. O., Giblet E. R., Baughan M. A., Hopkinson D. A., Povey S., Harris H., r968. Inherited variations in human phosphohexose-isomerase. Ann. hum. Genet., 31, 329-338.

Fagerhol M. K., 1967. Serum Pi types in Norwegians. Acta Pathol. Microbiol. Scand., 70, 42 I-428.

Fildes R. A., PARr C. W., I963. Human red cell phosphogluconate dehydrogenase. Nature, 200, $890-891$. 
Gahne B., 1966. Studies on the inheritance of the electrophoretic forms of transferrins, albumins, prealbumins and plasma esterases of horses. Genetics, 53, 68ז-694.

Hopkinson D. A., Spencer N., Harris H., ig63. Red cell acid phosphatase variauts : a new human polymorphism. Nature, 199, 969 .

KAminski M., I969. Species specific and common esterases of equidae. I. Horse and donkey. Biochem. Biophys. Acta, 191, 6r I-620.

Kaminski M., Podliachouk L., I97o. Serum esterases of equidae : Truly or apparently negative phenotypes. Comp. Biochem. Physiol., 36, 207-209.

Kaminski M., Podliachouk L., Vandeplassche M., Girard O., I97o. Ontogenetic variation of serum esterases in the horse. Z Z bl. Vet. Med. A, 17, 719-725.

Kelly E., Stormont C., Suzuki Y., I97I. Catalase polymorphism in the red cells of horses. Anim. Blood Grps. Biochem. Genet., 2, I35-I43.

Podliachouk L., Vandeplassche M., Bouters R., I974. Gestation gemellaire, chimerisme et freemartinisme chez le cheval. Acta Zool. Biol. Antwerpiensia (in press).

SANDBERG K., I968. Genetic polymorphism in carbonic anhydrase from horse erythrocytes. Hereditas, 60, 4II-4I2.

SAndberg K., Bengtsson S., 1972. Polymorphism of hemoglobin and 6-phosphogluconate dehydrogenase in horse erythrocytes. Proc. 12th Eur. Conf. Anim. Blood Grps, Biochem. Polymorph. Budapest, 527-531.

Shaw D. H., Stone W. H., 1962. Time of appearance of antigenic factors on cattle erythrocytes. Proc. Soc. Exp. Biol. Med., 111, I04-III.

Scotт A. M., I972. Improved separation of polymorphic esterases in horses. Proc. 12th Eur. Conf. Anim. Blood Grps, Biochem. Polymorph. Budapest, 55 I-553.

Spencer N., Hopkinson D. A., Harris H., i964. Phosphoglucomutase polymorphism in man. Nature, 204, 742-745.

Stormont C., Suzuki Y., 1963. Genetic control of Albumin phenotypes in horses. Proc. Soc. Exp. Biol. Med., 114, 673-675.

TAPPAN D. V., JaceY M. J., Boyden H. M., 1964. Carbonic anhydrase isozymes of neonatal and adult human and some animal erythrocytes. In "Gel electrophoresis ". Ann. N. Y. Acad. Sci., 121, 589-599. 